\title{
Examining Motivations and Self-regulated Learning Strategies of Returning MOOCs Learners
}

\author{
Bodong Chen \\ University of Minnesota \\ Minneapolis, USA, 55455 \\ chenbd@umn.edu
}

\author{
Yizhou Fan \\ Peking University \\ Beijing, China, 100871 \\ yizhou0034@126.com \\ Qiong Wang \\ Peking University \\ Beijing, China, 100871 \\ wangqiong@pku.edu.cn
}

\author{
Guogang Zhang \\ Beijing Institute of Technology \\ Beijing, China, 100871 \\ yhhzgg@163.com
}

\begin{abstract}
The present study examines behavioral patterns, motivations, and self-regulated learning strategies of returning learners - a special learner subpopulation in massive open online courses (MOOCs). To this end, data were collected from a teacher professional development MOOC that has been offered for seven iterations during 2014-2016. Data analysis identified more than $15 \%$ of all registrants as returning learners. Findings from click log analysis identified possible motivations of re-enrollment including improving grades, refreshing theoretical understanding, and solving practical problems. Further analysis uncovered evidence of self-regulated learning strategies among returning learners. Taken together, this study contributes to ongoing inquiry into MOOCs learning pathways, informs future MOOC design, and sheds light on the exploration of MOOCs as a viable option for teacher professional development.
\end{abstract}

\section{CCS Concepts}

-Applied computing $\rightarrow$ Distance learning; •Information systems $\rightarrow$ Clustering;

\section{Keywords}

MOOCs; teacher professional development; clustering

\section{INTRODUCTION}

Massive open online course (MOOC) research has evolved to recognize various pedagogical models, varied conceptions of learner engagement and success [1, 2], and different learner subpopulations with varied learner intentions [3]. However, so far little work has examined returning learners - a special learner subpopulation who keep returning to a same MOOC after initial attempts. One study in this area finds

Permission to make digital or hard copies of part or all of this work for personal or classroom use is granted without fee provided that copies are not made or distributed for profit or commercial advantage and that copies bear this notice and the full citation on the first page. Copyrights for third-party components of this work must be honored. For all other uses, contact the owner/author(s).

LAK '17 March 13-17, 2017, Vancouver, BC, Canada

(C) 2017 Copyright held by the owner/author(s).

ACM ISBN 978-1-4503-4870-6/17/03.

DOI: http://dx.doi.org/10.1145/3027385.3029448 that while MOOC learners commonly re-take a course for eventual completion, some learners re-take a MOOC despite success in earlier trials [7]. Motivations behind returning learners in MOOCs are thus worth further investigation.

Returning learners are especially worth attention in MOOCs designed for teacher professional development (PD) in developing regions. While traditional teacher $\mathrm{PD}$ follows the form of one-shot workshops that emphasize information delivery and fall short on catalyzing classroom changes [6], course re-taking in teacher $\mathrm{PD}$ MOOCs demonstrates opportunities for sustained PD integrated in teachers' daily practice.

This poster reports early findings from a project investigating MOOCs as a potential option for teacher $\mathrm{PD}$ in underdeveloped regions of China. By focusing on returning learners, this project attempts to address the following research questions: (1) What were the general demographics of returning learners; (2) What were the potential motivations of their re-enrollment; and (3) To which extent had they demonstrated self-regulated learning strategies.

\section{METHODS}

\subsection{Context}

The research context was one teacher PD MOOC, titled Flipped Classroom, offered for seven times during July 2014 and March 2016 by the X-Learning Center of Peking University. As learners were free to enroll in each offering, this MOOC provided a unique opportunity for investigating returning learners over a long period of time. During all seven iterations, a total of 126,044 learners registered, with K-12 and higher education teachers representing more than $80 \%$ of them.

\subsection{Data and analyses}

To study returning learners in this MOOC, we collected a wide range of data including entry-surveys, course enrollment data, course click logs, and learner performance data.

To answer research questions, we first identified returning learners based on course enrollment data. Descriptive analysis of their responses to entry-surveys were used to depict a general picture of their demographics. Click logs and performance data were mined to address the other questions.

\section{RESULTS}




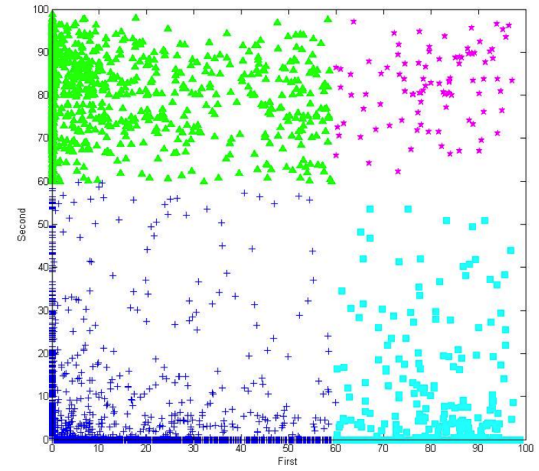

Figure 1: Two time learners' course grades.

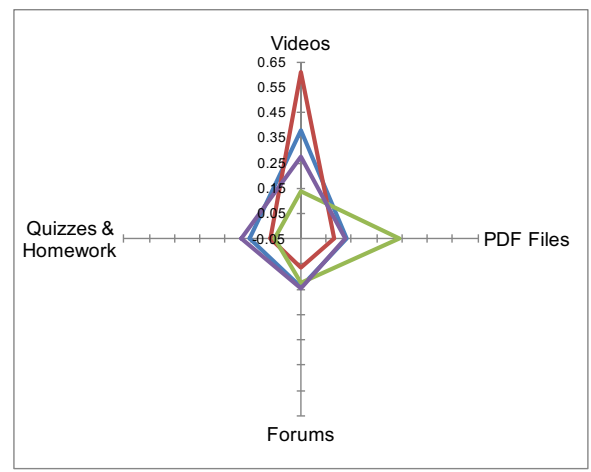

Figure 2: Clustering of returning learners.

\subsection{Basic demographics of returning learners}

Results indicated that more than $15 \%$ of all registrants in this MOOC were returning learners, with a median age of 35 and $65.69 \%$ females. More than $80 \%$ of all returning learners were teachers, including K-6 teachers (15.72\%), middle-high school teachers $(33.70 \%)$, and college teachers $(31.38 \%)$. The geographic locations covered all provinces of Mainland China.

\subsection{Possible motivations of re-enrollment}

As improving performance is a possible motivation for re-enrollment [7], we first analyzed the changes of course grades between two enrollments of two-time course takers. As shown in Figure 1, the green cluster achieved substantially higher score at their second attempt, while the light blue cluster showed less interests in improving scores as they had already passed the course at their prior attempts. We speculate that returning learners in the green cluster were driven by better grades, and those in the light blue cluster were driven by emerging learning needs in their teaching.

\subsection{Self-regulated learning strategies}

Connected with learner motivations were self-regulated learning (SRL) strategies in MOOC learning such as planning and controlling the learning process [4]. To uncover SRL strategies from the angle of selective course engagement, we derived from click logs several variables based on learners' use of course resources (e.g., videos, PDF handouts, forums) and conducted cluster analysis to distinguish subgroups in returning learners. Results identified four clusters, characterized as video viewers (red), handout collectors (green), strivers (violet), and others (blue; see Figure 2). Connected with potentially diversified motivations [5], returning learners demonstrated selective course engagement leading to varied focus on course resources.

Since video viewing was salient among all clusters except for handout collectors, we further conducted association rule mining (using the Apriori algorithm) to identify videos that were most frequently watched by returning learners at their second learning attempts. Results uncovered their interests in course videos covering key learning theories (e.g., Bloom's Taxonomy) and practical guidance on flipped classroom (e.g., screen-casting with Camtasia). Their interests in reviewing those videos implied areas returning learners could use additional support.

\section{CONCLUSIONS}

To conclude, this preliminary study attempted to study returning learners - an underexplored learner subpopulation in MOOCs - and uncovered their potentially diverse motivations and SRL strategies related to selective engagement. Ongoing efforts aim to deepen these analyses and seek further explanations through qualitative inquiry.

\section{ACKNOWLEDGMENTS}

This research was funded by the Digital Learning for Development (DL4D) initiative in Asia. Grant No. 2016-0002.

\section{REFERENCES}

[1] L. B. Breslow and D. E. Pritchard. Studying learning in the worldwide classroom: Research into edx's first MOOC. Research \& Practice Assessment, 8(1):13-25, 2013.

[2] J. DeBoer, A. D. Ho, G. S. Stump, and L. Breslow. Changing "course": Reconceptualizing educational variables for massive open online courses. Educational researcher, 43(2):74-84, Feb. 2014.

[3] R. Ferguson and D. Clow. Examining engagement: Analysing learner subpopulations in massive open online courses (MOOCs). In Proceedings of the Fifth International Conference on Learning Analytics And Knowledge - LAK '15, pages 51-58, New York, New York, USA, 2015. ACM Press.

[4] R. F. Kizilcec, M. Pérez-Sanagustín, and J. J. Maldonado. Self-regulated learning strategies predict learner behavior and goal attainment in massive open online courses. Computers \& education, 104:18-33, 2017.

[5] R. F. Kizilcec and E. Schneider. Motivation as a lens to understand online learners: Toward Data-Driven design with the OLEI scale. ACM Trans. Comput. -Hum. Interact., 22(2):6:1-6:24, Mar. 2015.

[6] G. Kleiman, M. A. Wolf, and D. Frye. The digital learning transition MOOC for educators: exploring a scalable approach to professional development. Technical report, Friday Institute for Educational Innovation, 2013.

[7] A. Woodgate, H. Macleod, A.-M. Scott, and J. Haywood. Differences in online study behaviour between sub-populations of MOOC learners. Educación XX1, 18(2), 12 June 2015. 